\title{
Avaliação do nível de conhecimento sobre diástase abdominal em mulheres no estado do Pará
}

\author{
Assessment of the level of knowledge about abdominal diastasis in women in the state of Pará \\ Evaluación del nivel de conocimientos sobre diástasis abdominal en mujeres del estado de Pará \\ Marina Silva Nicolau Taketomi ${ }^{1 *}$, Amanda Miguel Santos ${ }^{1}$, Juciele Rodrigues de Aguiar ${ }^{1}$.
}

\begin{abstract}
RESUMO
Objetivo: Avaliar o nível de conhecimento das mulheres a respeito da diástase do músculo reto abdominal após a gestação. Métodos: Para a obtenção dos dados ocorreu a aplicação de questionário via internet através de uma plataforma online, com mulheres entre 18 e 60 anos de idade, residentes da região Paraense. Resultados: Obteve-se um N amostral de 100 mulheres, sendo que $80 \%$ destas apresentavam idade entre 18 e 30 anos e $58 \%$ da amostra apontaram ter Ensino Superior Incompleto. Das componentes, 32\% tiveram alguma gestação, e entre estas, apenas 3,2\% realizaram tratamento fisioterapêutico durante este período. $54 \%$ das voluntárias afirmam saber o que é diástase abdominal, entretanto somente $35 \%$ das participantes informaram ter conhecimento sobre a atuação da fisioterapia no tratamento da diástase. Conclusão: Os dados encontrados demonstram carência na difusão de conhecimento sobre a DMRA após a gestação e do tratamento fisioterapêutico para esta condição, tais fatos apontam íntima relação com o baixo nível de escolaridade das mulheres da amostra e baixa disseminação de informaçõ̉es sobre a atuação dos profissionais fisioterapeutas pélvicos entre a comunidade pesquisada.
\end{abstract}

Palavras chaves: Diástase muscular, Gravidez, Período pós-parto, Fisioterapia.

\section{ABSTRACT}

Objective: To evaluate the level of knowledge of women about diastasis of the rectus abdominis muscle after pregnancy. Methods: To obtain the data, a questionnaire was applied via the Internet through an online platform, with women between 18 and 60 years of age, residents of the Paraense region. Results: A sample $\mathrm{N}$ of 100 women was obtained, $80 \%$ of whom were aged between 18 and 30 years and $58 \%$ of the sample indicated they had incomplete higher education. Of the components, $32 \%$ had some pregnancy, and among these, only 3.2\% underwent physical therapy during this period. $54 \%$ of the volunteers claim to know what abdominal diastasis is, however only $35 \%$ of the participants reported having knowledge about the role of physiotherapy in the treatment of diastasis. Conclusion: The data found show a lack of knowledge about DMRA after pregnancy and physiotherapeutic treatment for this condition, such facts point to a close relationship with the low level of education of the women in the sample and low dissemination of information about the performance of professional's pelvic physiotherapists among the researched community.

Keywords: Muscle diastasis, Pregnancy, Postpartum period, Physiotherapy.

RESUMEN

Objetivo: Evaluar el nivel de conocimiento de las mujeres sobre la diástasis del músculo recto del abdomen después del embarazo. Métodos: Para la obtención de los datos se aplicó un cuestionario vía Internet a través de una plataforma online, con mujeres entre 18 y 60 años, residentes de la región de Paraense. Resultados: Se obtuvo una muestra N de 100 mujeres, el 80\% de las cuales tenían entre 18 y 30 años y el $58 \%$ de la muestra indicó tener estudios superiores incompletos. De los componentes, el $32 \%$ tuvo algún embarazo, y entre estos, solo el 3,2\% se sometió a fisioterapia durante este período. El $54 \%$ de los voluntarios afirma saber qué es la diástasis abdominal, sin embargo, solo el $35 \%$ de los participantes informó tener conocimiento sobre el papel de la fisioterapia en el tratamiento de la diástasis. Conclusión: Los datos encontrados evidencian un desconocimiento sobre DMRA post embarazo y tratamiento fisioterapéutico para esta condición, tales hechos apuntan a una estrecha relación con el bajo nivel de educación de las mujeres de la muestra y la baja difusión de información sobre el desempeño de los profesionales. fisioterapeutas pélvicos entre la comunidad investigada.

Palabras clave: Diástasis muscular, El embarazo, Período pós-parto, Fisioterapia.

${ }^{1}$ Universidade do Estado do Pará (UEPA), Santarém - PA. *E-mail: taketomi@professor.iespes.edu.br 


\section{INTRODUÇÃO}

A Diástase do Músculo Reto Abdominal (DMRA) é uma condição em que ocorre a modificação do posicionamento fisiológico dos músculos retos do abdômen, ocasionando a separação dos ventres musculares, independentemente da localização desta separação em relação a cicatriz umbilical, desde que ocorra ao longo da linha alba, esse estiramento ocorre devido à distensão abdominal esperada na gravidez (MOTA P, et al., 2015).

A progesterona, estrogênio e relaxina são alguns dos hormônios que influenciam as mudanças fisiológicas e anatômicas do corpo gravídico. Dentre estes, a relaxina é o hormônio responsável pelo aumento da mobilidade articular pelo relaxamento dos ligamentos e articulações do corpo, de maneira a acompanhar o crescimento uterino e facilitar o parto por via vaginal (DERMARTINI E, et al., 2016). O crescimento do feto, a inclinação pélvica anterior com ou sem hiperlordose lombar, o aumento da pressão intra-abdominal e o efeito da relaxina são fatores que contribuem para o desenvolvimento da diástase dos músculos reto abdominais (VELJOVIC F, et al., 2019; MICHALSKA A, et al., 2018).

A DMRA apresenta-se mais evidente no último trimestre de gravidez, entretanto pode aparecer durante 0 trabalho de parto em consequência do esforço físico realizado para a expulsão do feto (LEITE AC e ARAÚJO KK, 2012). Entre mulheres que estão no período do puerpério (pós-parto), as consequências relatadas na literatura sobre DMRA são alterações na aparência abdominal, dor na região do abdômen, dor lombar, dor pélvica e queixas uroginecológicas (KESHWANI N, et al., 2018).

Entre as complicações mais comuns da DMRA está a incontinência urinária (CARVALHO AMP, et al., 2014). A mesma ocorre devido o afastamento dos músculos reto abdominais, que interferem no assoalho pélvico, devido a diminuição da pressão de retenção destes e consequente aumento da pressão de expulsão, causando assim a perda de urina de forma involuntária, principalmente durante momentos de esforço, como tossir, carregar peso, espirar, entre outros (LUNA DCB, et al., 2012).

Além das referidas complicações, quando ocorre diástase durante o período gestacional há maior exposição do feto. A ocorrência dessa fragilidade se dá pela não proteção da região pela camada muscular abdominal podendo ocasionar possíveis complicações (URBANO FA, et al., 2019).

É necessário que haja instruções adequadas em relação ao tratamento da DMRA, principalmente em casos acompanhados por hérnia umbilical, pois o mesmo poderá sofrer alterações visando tratar não apenas a diástase, mas também todas as outras alterações corporais e sistêmicas que ocorreram em decorrência dessa condição (VELJOVIC F, et al., 2019).

A fisioterapia dispõe de recursos essenciais para a eliminação da DMRA como, eletroterapia, cinesioterapia, pilates e hidroterapia. Essas técnicas apresentam eficácia comprovada por meio de estudos clínicos que evidenciam a importância do profissional fisioterapeuta no campo da Saúde da Mulher na atenção à gestante. Entretanto, faz-se notório a insuficiência de estudos abordando as intervenções fisioterapêuticas para prevenção e tratamento da diástase gestacional (COITINHO LMF, et al., 2019).

Levando em consideração que a maioria dos estudos publicados investigam a ocorrência, mensuração e tratamento da DMRA e, portanto, percebeu-se a necessidade de pesquisar o grau de informação entre as mulheres sobre este assunto tão importante. Assim, o presente artigo tem como objetivo avaliar o nível de conhecimento das mulheres a respeito da diástase do músculo reto abdominal após a gestação.

\section{MÉTODOS}

A pesquisa foi realizada por meio de um formulário em uma plataforma online. O estudo levou em consideração os aspectos éticos da Resolução 466/2012 do Conselho Nacional de Saúde (CNS) que regulariza as pesquisas que envolvam seres humanos e foi aprovado pelo CEP da Universidade do Estado do Pará, campus XII, CAAE 42371321.1.0000.5168.

Ressalta-se que os dados coletados apenas foram catalogados a partir da autorização legal das participantes em que a análise foi realizada, mantendo o anonimato das mesmas e a confidencialidade das informações obtidas. Com este intuito, foram utilizados códigos numéricos a fim de evitar possíveis exposições dos envolvidos na pesquisa e as pesquisadoras são responsáveis por assegurar que todas as informações foram armazenadas em banco de dados particular e após 5 anos serão deletados e incinerados. 
A coleta de dados ocorreu em três etapas principais: primeiramente, houve a divulgação da pesquisa em redes sociais pessoais, afim de informar as mulheres sobre a pesquisa que seria realizada e convidá-las a participar; logo após, as interessadas em participar na pesquisa receberam o link do formulário online, contendo o Termo de Consentimento Livre e Esclarecido (TCLE) e a pesquisa de forma individual; e para finalizar, as voluntárias que concordaram com o TCLE, na Terceira etapa realizaram o preenchimento do questionário elaborado pelas autoras, de acordo com aquilo que conheciam.

O período da coleta de dados ocorreu entre os meses de março e abril de 2021. Os critérios de inclusão foram: mulheres, entre 18 a 60 anos que tenham acesso ao serviço de internet e aceitem participar, de forma voluntária, na pesquisa. Os critérios de exclusão foram: mulheres sem acesso à internet e mulheres que responderam menos de $80 \%$ do questionário proposto.

Após a coleta de dados, as informações foram organizadas em uma planilha no Microsoft Excel, e verificados dados de média, mediana e desvio padrão, para posterior análise e comparação dos resultados.

\section{RESULTADOS}

Ao final da aplicação do formulário online, obteve-se o $\mathrm{N}$ amostral de 100 mulheres. A seguir há três tabelas que resumem os dados coletados, sendo divididas entre: Perfil sociodemográfico, perfil obstétrico e perfil do conhecimento a respeito da Diástase dos Músculos Reto Abdominais das participantes voluntárias da pesquisa no Pará.

Das 100 mulheres analisadas, 80\% têm média de 18 a 30 anos, $13 \%$ apresentam média de 31 a 40 anos, $4 \%$ têm média de 41 a 50 anos, $1 \%$ têm média de 51 a 60 anos e $2 \%$ não responderam a idade. Sendo que 94\% das voluntárias são residentes do município de Santarém no Estado do Pará, 1\% reside no município de Belém no Estado do Pará e 5\% residentes em demais localidades. Entre as participantes da pesquisa, 22\% têm o Ensino Superior completo, 58\% apresentam Ensino Superior incompleto, 17\% têm Ensino Médio completo e 3\% apresentam Ensino Médio incompleto (Tabela 1).

Tabela 1 - Perfil sociodemográfico das participantes voluntárias da pesquisa no Pará.

\begin{tabular}{lcc}
\hline Características & N (número total) & $\%$ \\
\hline Sexo & & \\
\hline Feminino & 100 & $100 \%$ \\
Masculino & 0 & \\
\hline Idade & & \\
\hline $18-30$ anos & 80 & $80 \%$ \\
$31-40$ anos & 13 & $13 \%$ \\
$41-50$ anos & 4 & $4 \%$ \\
$51-60$ anos & 1 & $1 \%$ \\
Não responderam & 2 & $2 \%$ \\
\hline Cidade & & \\
\hline Santarém & 94 & $94 \%$ \\
Belém & 1 & $5 \%$ \\
Outras & 5 & \\
\hline Nível de Escolaridade & & $22 \%$ \\
\hline Ensino Superior Completo & 22 & $17 \%$ \\
Ensino Superior Incompleto & 58 & $3 \%$ \\
Ensino Médio Completo & 17 & \\
Ensino Médio Incompleto & 3 & \\
\hline
\end{tabular}

Fonte: Taketomi MSN, et al., 2021. 
Das mulheres analisadas na pesquisa, 68\% eram nulíparas, $11 \%$ primíparas e $21 \%$ eram multíparas. Entre as 100 mulheres voluntárias, $12 \%$ tiveram um parto, $13 \%$ tiveram dois partos, $5 \%$ obtiveram três partos e $2 \%$ tiveram quatro ou mais partos. Dos tipos de parto, $60 \%$ foram cesarianos e $40 \%$ foram normais (Tabela 2 ).

No caso das participantes que declararam pelo menos uma gestação, 65,6\% relataram que praticavam atividade física durante a gravidez e 34,4\% não realizaram atividade física durante esse período. Entre estas, $3,2 \%$ relatou ter acompanhamento fisioterapêutico durante a gestação e $96,8 \%$ informou que não houve acompanhamento de um profissional fisioterapeuta (Tabela 2).

Tabela 2 - Perfil obstétrico das participantes voluntárias da pesquisa no Pará.

\begin{tabular}{lcc}
\hline Características & N (número total) & $\%$ \\
\hline Número de gestações & 68 & $68 \%$ \\
\hline Nulípara & 11 & $11 \%$ \\
Primípara & 21 & $21 \%$ \\
Multípara & & \\
\hline Números de Partos & 68 & $68 \%$ \\
\hline Nenhum & 12 & $12 \%$ \\
Um & 13 & $13 \%$ \\
Dois & 5 & $5 \%$ \\
Três & 2 & $2 \%$ \\
Quatro ou mais & & $60 \%$ \\
\hline Tipos de Parto & 21 & $40 \%$ \\
\hline Cesária & 14 & \\
Normal & & $65,60 \%$ \\
\hline Realizou Atividade Física Durante a Gravidez & 21 & $34,40 \%$ \\
\hline Sim & 11 & $3,20 \%$ \\
Não & 1 & $96,80 \%$ \\
\hline Durante sua gestação você teve acompanhamento fisioterapêutico &
\end{tabular}

Fonte: Taketomi MSN, et al., 2021.

Entre as 100 mulheres analisadas, 54\% delas afirmam saber o que é diástase abdominal, 43\% não sabiam o que é e 3\% não responderam. 20\% dizem conhecer a classificação da diástase, $75 \%$ não sabem classificar a mesma e $5 \%$ não responderam. $24 \%$ das participantes declaram que sabem dizer se apresentam diástase ou não, porém $68 \%$ não sabem se tem e $8 \%$ deram resposta.

Das voluntárias, $58 \%$ sabem que existe tratamento para a diástase abdominal, 38\% declararam não saber informar e 4\% não responderam. Dentre as voluntárias que apresentaram DMRA, 3\% realizaram tratamento para esta; $20 \%$ declarou que não realizou tratamento, $53 \%$ não sabiam informar se tiveram diástase e $24 \%$ não responderam.

Em relação as mulheres que apresentaram diástase e realizaram tratamento, duas o fizeram com um profissional fisioterapeuta e uma com um médico. 35\% das participantes dizem conhecer o tratamento fisioterapêutico para a DRMA, entretanto 57\% alegam não conhecer e $8 \%$ não responderam (Tabela 3). 
Tabela 3 - Perfil do conhecimento a respeito da Diástase dos Músculos Reto Abdominais das participantes voluntárias da pesquisa no Pará.

\begin{tabular}{|c|c|c|}
\hline Características & $\mathbf{N}$ (número total) & $\%$ \\
\hline \multicolumn{3}{|c|}{ Você sabe o que é Diástase Abdominal } \\
\hline Sim & 54 & $54 \%$ \\
\hline Não & 43 & $43 \%$ \\
\hline Não responderam & 3 & $3 \%$ \\
\hline \multicolumn{3}{|c|}{ Conhece as classificações da diástase } \\
\hline Sim & 20 & $20 \%$ \\
\hline Não & 75 & $75 \%$ \\
\hline Não responderam & 5 & $5 \%$ \\
\hline \multicolumn{3}{|l|}{ Você sabe se tem diástase } \\
\hline Sim & 24 & $24 \%$ \\
\hline Não & 68 & $68 \%$ \\
\hline Não responderam & 8 & $8 \%$ \\
\hline \multicolumn{3}{|c|}{ Você sabe se existe tratamento para diástase } \\
\hline Sim & 58 & $58 \%$ \\
\hline Não & 38 & $38 \%$ \\
\hline Não responderam & 4 & $4 \%$ \\
\hline \multicolumn{3}{|c|}{ Se você já teve diástase, realizou algum tratamento para ela? } \\
\hline Sim & 3 & $3 \%$ \\
\hline Não & 20 & $20 \%$ \\
\hline Não sei se tenho diástase & 53 & $53 \%$ \\
\hline Não responderam & 24 & $24 \%$ \\
\hline \multicolumn{3}{|c|}{ Se sim, qual profissional fez teu tratamento? } \\
\hline Fisioterapeuta & 2 & $66,70 \%$ \\
\hline Médico & 1 & $33,30 \%$ \\
\hline Esteticista & 0 & $0 \%$ \\
\hline Educador Físico & 0 & $0 \%$ \\
\hline \multicolumn{3}{|c|}{ Você conhece o tratamento fisioterapêutico para diástase abdominal } \\
\hline Sim & 35 & $35 \%$ \\
\hline Não & 57 & $57 \%$ \\
\hline Não responderam & 8 & $8 \%$ \\
\hline
\end{tabular}

Fonte: Taketomi MSN, et al., 2021.

\section{DISCUSSÃO}

Foi observado que a maioria das participantes residiam no município de Santarém no Estado do Pará. Averiguando os dados notou-se que em relação a sapiência sobre o que se tratava a diástase abdominal, as participantes que mais responderam saber sobre esta condição tinham entre 18 e 30 anos, esse achado pode ser justificado em decorrência de que a grande parte das respostas recebidas foram desta mesma faixa etária. Em relação à escolaridade das participantes que informaram saber o que é DRMA, a maioria das respostas foram de mulheres com Ensino Superior incompleto, seguido de voluntárias que completaram o Ensino Superior.

Entre participantes que alegaram não terem conhecimento do termo e seu significado, notou-se maior incidência em mulheres com Ensino Médio completo e incompleto. Tais dados sobre conhecimento e 
desconhecimento da DMRA, demonstram significativa correlação com o nível de escolaridade das voluntárias. Reafirmando dado apontado pelo Ministério da Saúde (2012) que identifica a baixa escolaridade como possível fator de risco para ocorrências de alterações na gravidez e puerpério, pois pode estar diretamente relacionada ao menor acesso à informação e a limitada compreensão da importância dos cuidados com a saúde tanto da mãe quanto do bebê.

Entre as 100 mulheres haviam 32 que já estiveram gestantes, sendo a porcentagem de partos do tipo normal correspondente a 48,8\% e partos de tipo cesariano com média de $51,2 \%$. Este achado concorda com Schutz PO e Porciuncula MB (2020), que observaram que 90,9\% das entrevistadas foram submetidas à cesariana, e elas foram levadas a esse método por vários motivos adversos, mesmo apesar de a maioria apresentar como preferência inicial a via de parto vaginal que seria justificado por fatores como amamentação precoce e recuperação pós-parto mais rápida.

Relacionado ao perfil obstétrico, pode-se observar que apesar da maioria das participantes que apresentaram pelo menos uma gravidez apontarem ter praticado atividades físicas durante a gestação, uma quantidade significativa não adotou a prática, concordando com Soares DSC, et al. (2017), onde afirma que embora os benefícios de realizar atividade física durante a gestação serem de conhecimento de muitas gestantes, a maioria não adere a este hábito, seja por restrição médica, receio, falta de profissional capacitado para acompanhar, entre outros motivos.

Analisando as mulheres que tiveram a pratica de exercícios no período gestacional se tornou possível mensurar que as atividades físicas mais praticadas por elas foram a caminhada e o método Pilates. Tais achados são justificados por Soares DSC, et al. (2017), que expões que a caminhada é uma das atividades mais recomendadas para gestantes, por ser uma prática de baixo impacto, e por Vasconcelos EH, et al. (2017), que também indica o método Pilates durante o período gestacional, o que corrobora com os pesquisadores Cota ME e Metzker CAB (2019) falam, sobre efeitos proveitosos desta técnica no alívio da dor lombar durante a gestação, porém os autores não afirmam que o método é eficaz para prevenção ou tratamento da DMRA.

Através da análise das respostas foi possível observar que parte considerável das mulheres, mesmo após o processo da gestação, não sabem do que se trata a DMRA, desta forma é notório que há escassez de informações sobre o assunto tanto para a população em geral, quanto para mulheres no período de gestação e puerperal. Tal achado torna-se evidente com a existência de grande quantidade de estudos científicos voltados apenas para aspectos clínicos da DMRA, e exorbitante escassez de trabalhos direcionados para a avaliação do conhecimento popular a respeito da temática.

Das 100 mulheres avaliadas, 54\% relatam saber o que é diástase abdominal. Quando houve o questionamento sobre o que elas entendiam ser a diástase abdominal, a maioria das voluntárias caracterizaram-na como sendo um afastamento ou separação dos músculos abdominais ocasionados principalmente na gravidez. Entretanto, uma pequena parte das voluntárias descreveram a diástase como sendo o "esticamento", "rompimento" e "distensão" do músculo abdominal, associando que a "barriga não volta ao normal” após o período gravídico.

Tais respostas evidenciam que o conhecimento sobre a DRMA não é completamente difundido entre a maioria das mulheres e mesmo entre as que relatam conhecimento do termo, nem todas sabem definir o exato significado deste, como consequência dessa falta de conhecimento é notória a baixa procura por tratamentos especializados durante a gestação e no pós-parto. Durante a pesquisa para produção do presente artigo, não foram encontrados estudos que abordam o nível de conhecimento das mulheres sobre a DMRA.

No presente estudo, foi encontrado que a maioria das voluntárias não detinham informação sobre a ocorrência da DMRA e informaram não conhecer a existência o tratamento fisioterapêutico para tal disfunção. Assim, nota-se carência de informações mais detalhadas em relação às mudanças e possíveis soluções para as alterações que ocorrem no corpo das gestantes e puérperas. Vasconcelos EH, et al. (2017), certifica a importância de proporcionar uma assistência obstétrica eficiente que abranja todo ciclo gravídico e puerperal, considerando as necessidades físicas, biológicas e psicossociais da gestante. 
Saraiva DSD, et al. (2019), Dermartini E, et al. (2016), Leite ACNMT e Araújo KKBC (2012) e Santos MD, et al. (2016), declaram que a DMRA é considerada de caráter patológico quando a medida de afastamento dos músculos reto-abdominais é igual ou superior a $3 \mathrm{~cm}$, que traz prejuízo a funcionalidade do organismo da mulher como, contenção visceral, defecação, estabilização da postura, movimentação de tronco e parto. Considera-se que a diástase é fisiológica ao apresentar de 12 a $15 \mathrm{~cm}$ de largura durante o período gestacional e retorna para até $3 \mathrm{~cm}$ após a gestação. Apesar dos achados citados, há controvérsias na literatura sobre os valores de padronização da DRMA.

Da amostra, seis mulheres apontaram ter DMRA. Referente ao tamanho da diástase apresentado por estas, uma componente informou ter $2 \mathrm{~cm}$, outra, $3 \mathrm{~cm}$ e duas informaram $1 \mathrm{~cm}$. As demais participantes não souberam mensurar o tamanho da DMRA que lhes ocorreu. Destas, três voluntárias relataram a realização de tratamento para a referida alteração, e entre estas, duas efetuaram o procedimento com fisioterapeuta e uma com médico. Durante o preenchimento do formulário para a presente pesquisa não foram questionados quais os critérios utilizados pelos profissionais para diagnosticar a presença da diástase abdominal e tratá-la.

Urbano FA, et al. (2019), aponta que durante o período gestacional, caso ocorra diástase, a mulher não relatará dores na região abdominal, entretanto, no período puerperal e pós-puerperal, esta alteração pode ocasionar complicações para a saúde da mulher, como dores na região lombar, hérnia umbilical e alterações uroginecológias.

Deenika $R$, et al. (2019) associou que quanto maior o afastamento dos músculos reto-abdominais apresentados, menor a força abdominal e maior o comprometimento e grau de dor na região lombar que será referido, haja vista que ocorre a mudança de origem e inserção fisiológica desta musculatura. Tais estudos corroboram com os achados de Pereira TRC, et al. (2017), em que há a associação a dor lombar no puerpério com a ocorrência de diástase dos músculos retos abdominais e também aponta o comprometimento na capacidade da musculatura abdominal de estabilização do tronco, alterando o alinhamento postural e o equilíbrio da musculatura da região lombar.

Entre as voluntárias que relataram ter tido diástase, a maioria apresentou perda urinária involuntária ao tossir, rir, carregar peso, e/ou casos de urgência miccional. Apenas uma voluntária mencionou a ocorrência de hérnia umbilical. Segundo os pesquisadores Franchi EF e Rahmeier $L$ (2016), os referidos sintomas são comumente associados a DRMA e merecem atenção e tratamento especializado.

Apenas 35\% das pesquisadas declarou conhecer o tratamento fisioterapêutico para DMRA. O pequeno número justifica-se pelo falo de poucas mulheres deterem conhecimento sobre o que é diástase e consequentemente, por não saberem do que se trata, não buscam maiores informações a respeito do tratamento desta alteração, assim como não conhecem as consequências funcionais da DMRA.

Fundamentado em Vasconcelos $\mathrm{EH}$, et al. (2017) constata-se que por intermédio do tratamento fisioterapêutico, haverá o favorecimento do retorno do posicionamento dos ventres musculares dos músculos reto abdominais ao estado pré-gravídico, auxiliando, assim no tratamento das disfunções ocasionadas pela diástase e na recuperação da imagem corporal positiva da mulher após a gestação. Este estudo também detectou que os recursos mais eficazes para a redução da DRMA são o uso da eletroterapia, cinesioterapia, do método pilates e também a pratica da hidroterapia.

A cinesioterapia é uma pratica exclusiva do profissional da fisioterapia e que este tem um papel fundamental para a recuperação da mulher durante o puerpério imediato, incluindo a DRMA (MICHELOWSKI ACS, et al., 2014). O que contribui com o achado de Feitosa GZ, et al. (2017), onde em sua revisão de literatura, conclui que o tratamento conservador através de modalidades que visam o fortalecimento muscular encontra resultados satisfatórios no tratamento da DMRA, reforça-se que mesmo em protocolos não convencionais a cinesioterapia é utilizada como recurso coadjuvante.

Os exercícios de fortalecimento quando associados com atividades resistidas e aeróbicas na DMRA são os mais indicados no período puerperal e/ou pós-puerperal. Além dessas técnicas, as sessões podem ser complementadas com a pratica de ginástica abdominal hipopressiva e a utilização de eletroestimulação que, segundo este estudo, favorecem o encurtamento do período de recuperação desta condição. $O$ mesmo 
também relata que os referidos exercícios são uma escolha de caráter excelente considerando as dificuldades enfrentadas durante o período do puerpério pelas parturientes, e que a melhora ou reversão do quadro de DMRA pode ser realizada sem necessidade de que haja intervenções cirúrgicas (URBANO FA, et al., 2019).

A eficiência dos tratamentos fisioterapêuticos para DRMA no puerpério realizando uma revisão de literatura com o tema proposto, onde foram analisados estudos publicados entre os anos de 2008 a 2018, ressaltando assim, a importância da presença do fisioterapia no atendimento multiprofissional tanto no decorrer da gestação quanto no puerpério, para evitar complicações e ajuda a gestante com os ajustes as alterações que aparecerem, além disso, são imprescindíveis a realização de mais estudos, evidências científicas e embasamentos teóricos sobre o assunto para amplificar o conhecimento da eficácia fisioterapêutica na redução da diástase dos músculos reto abdominais após a gestação (COITINHO LMF, et al., 2019).

\section{CONCLUSÃO}

O conhecimento sobre a DMRA que ocorre após a gestação e seu tratamento fisioterapêutico não é amplamente difundido entre as mulheres paraenses. Tal fato demonstra íntima relação com o baixo nível de escolaridade apresentado pela amostra, possivelmente associado ao menor acesso a informações por estas mulheres e também pela baixa disseminação de informações referentes a atuação dos fisioterapeutas pélvicos entre a comunidade pesquisada. Com isso, sugere-se a ampliação das ações de educação em saúde para as mulheres, assim como estratégias de políticas públicas que favoreçam o acesso dessas mulheres as informações e aos tratamentos durante o período gravídico e puerperal.

\section{REFERÊNCIAS}

1. BRASIL. Ministério da Saúde. Secretaria de Atenção à Saúde. Departamento de Atenção Básica. Atenção ao prénatal de baixo risco. Brasília: Ministério da Saúde, 2012. (Série A. Normas e Manuais Técnicos. Cadernos de Atenção Básica, n³2).

2. CARVALHO AMP, et al. O impacto da incontinência urinária e seus fatores associados em idosos. Rev. Bras. Geriatr. Gerontol; Rio de Janeiro, 2014.

3. COTA ME, METZKER CAB. Efeitos do método pilates sobre a dor lombar em gestantes: uma revisão sistemática. Revista Ciência e Saúde On-line, 2019; 4(2).

4. COITINHO LMF, et al. Eficiência dos tratamentos fisioterapêuticos para a diástase do músculo reto abdominal no puerpério: uma revisão integrativa. RECHST - Edição 2019; 8(1): 39-52.

5. DEENIKA R, et al. Relationship between diastasis of the rectus abdominis muscle (DRAM) and musculoskeletal dysfunctions, pain and quality of life: a systematic review. Physiotherapy 105, 2019.

6. DEMARTINI E, et al. Diastasis of the rectus abdominis muscle prevalence in postpartum. Fisioter. mov., Curitiba, 2016; 29(2): 279-286.

7. FEITOSA GF, et al. Intervenção fisioterapêutica no tratamento da diástase abdominal pós-parto: uma revisão de literatura. Ciências Biológicas e de Saúde Unit, Alagoas, 2017; 4(2): 239-250.

8. FRANCHI EF, RAHMEIER L. Efeitos da ginástica abdominal hipopressiva no puerpério imediatoEstudos de casos. Cinergis, Santa Cruz do Sul. 2016; 17(2): 108-12.

9. KESHWANI N, et al. Relationship Between Interrectus Distance and Symptom Severity in Women with Diastasis Recti Abdominis in the Early Postpartum Period. Phys Ther. 2018; 98(3): 182-190.

10. LEITE ACNMT e ARAÚJO KKBC. Diástase dos retos abdominais em puérperas e sua relação com variáveis obstétricas. Fisioter Mov. 2012; 25(2): 389-97.

11. LUNA DCB, et al. Frequência da Diástase Abdominal Em Puérperas E Fatores De Risco Associados. Fisioterapia e Saúde Funcional, 2012; 1(2).

12. MICHELOWSKI ACS, et al. A eficácia da cinesioterapia na redução da diástase do músculo reto abdominal em puérperas de um hospital público em Feira de Santana-BA. Revista Brasileira de Saúde Funcional, 2014; $1(2): 5-5$.

13. MICHALSKA A, et al. Diastasis recti abdominis - a review of treatment methods. Ginekol Pol. 2018; 89(2): 97-101.

14. MOTA $P$, et al. Diastasis recti abdominis in pregnancy and postpartum period. risk factors, functional implications and resolution. Current women's health reviews, 2015; 11(1): 59-67.

15. PEREIRA TRC, et al. Existe associação entre os desconfortos no puerpério imediato e a via de parto? Um estudo observacional. ABCS Health Sci., 2017; 42(2): 80-84.

16. SANTOS MD, et al. Does abdominal diastasis influence lumbar pain during gestation? Rev Dor. 2016; 17(1): 43-6. 
17. SARAIVA DSD, et al. AMORIM, Avaliação da Diástase do Reto Abdominal e a Presença de Incontinência Urinária em Puérperas. Rev.Mult. Psic., 2019; 13(48): 292-300.

18. SCHUTZP PO, PORCIUNCULA MB (2020). Percepção de puérperas sobre a escolha da via de parto em um hospital da serra do Rio Grande do Sul. Revista Eletrônica Acervo Saúde, 2020: e2415.

19. SOARES DSC, et al. Atividade física na gestação: uma revisão integrativa. Revista Perspectiva: Ciência e Saúde, $2017 ; 2(2)$.

20. URBANO FA, et al. Exercícios de fortalecimento para o músculo reto abdominal como tratamento da diástase pósgestacional. Revista Ciência e Saúde On-line, 2019; 4(1).

21. VASCONCELOS EH, et al. A intervenção fisioterapêutica na diástase do musculo reto abdominal (DMRA). São PauloSP: Revista Saberes, 2017; 6: 1-13.

22. VELJOVIC F, et al. Spinal Column and Abdominal Muscles Loading in Pregnant Women Dependent on Working Postures. Acta Inform Med. 2019; (1): 54-57. 